Ophthalmologe 2020 $\cdot 117: 652-658$ https://doi.org/10.1007/s00347-020-01162-x Online publiziert: 1. Juli 2020

(c) Springer Medizin Verlag GmbH, ein Teil von Springer Nature 2020

\section{Hintergrund}

Die SARS-CoV-2(Severe Acute Respiratory Syndrome Coronavirus 2)-Pandemie hat während der ersten Monate des Jahres 2020 weltweit $\mathrm{zu}$ tiefgreifenden Veränderungen der medizinischen Versorgung mit massiven Einschränkungen bei chirurgischen Eingriffen und nichtdringlichen ambulanten wie stationären Behandlungen geführt [1-4]. Es wird geschätzt, dass weltweit und fächerübergreifend allein in den ersten 12 Wochen der Pandemie 28.404.603 Operationen, d.h. 2.367.050 Operationen pro Woche aufgrund von COVID-19 (CoronavirusKrankheit-2019) abgesagt wurden [5].

Im Zuge der Umstellung der Kliniken auf die zu erwartende Versorgung von COVID-19-Patienten mit Bereitstellung möglichst großer Intensivbettenkapazitäten und Beatmungsplätzen ist es dabei v. a. zu erheblichen Einschränkungen bei der Durchführung elektiver Eingriffe gekommen $[6,7]$. Aufgrund der dadurch entstandenen konkurrierenden Situation um stationäre Behandlungsplätze sowie

Die Mitglieder der Vereinigung Ophthalmologischer Lehrstuhlinhaber werden am Beitragsende in $\bullet$ Tab. 1 gelistet.

Lars-Olof Hattenbach ${ }^{1,2,3} \cdot$ Thomas Reinhard $^{2,4,6} \cdot$ Peter Walter $^{5,6}$.

Johannes Roider ${ }^{6,7} \cdot$ Nicolas Feltgen ${ }^{2,8,9} \cdot$ Lutz Hesse $^{2,3,10}$. Jens Schrecker ${ }^{3,11}$. Nicole Eter ${ }^{2,6,12} \cdot$ Vereinigung Ophthalmologischer Lehrstuhlinhaber

${ }^{1}$ Augenklinik, Klinikum Ludwigshafen, Ludwigshafen, Deutschland

${ }^{2}$ für die Deutsche Ophthalmologische Gesellschaft (DOG), München, Deutschland

${ }^{3}$ für die Vereinigung Deutscher Ophthalmologischer Chefärzte (DOCH), Glauchau, Deutschland

${ }^{4}$ Augenklinik, Universitätsmedizin Freiburg, Freiburg, Deutschland

${ }^{5}$ Augenklinik, Universitätsklinikum Aachen, Aachen, Deutschland

${ }^{6}$ für die Vereinigung Ophthalmologischer Lehrstuhlinhaber (VOL), Münster, Deutschland

${ }^{7}$ Augenklinik, Universitätsmedizin Schleswig-Holstein, Campus Kiel, Kiel, Deutschland

${ }^{8}$ Augenklinik, Universitätsmedizin Göttingen, Göttingen, Deutschland

${ }^{9}$ für die Deutsche Retinologische Gesellschaft, Freiburg, Deutschland

${ }^{10}$ Augenklinik, Klinikum Heilbronn, Heilbronn, Deutschland

${ }^{11}$ Augenklinik, Rudolf Virchow Klinikum Glauchau, Glauchau, Deutschland

${ }^{12}$ Universitäts-Augenklinik Münster, Münster, Deutschland

\title{
Krisenstrategien der Kliniken während der Pandemie
}

personelle und operative Ressourcen waren bettenführende Augenkliniken, insbesondere an Häusern der Maximalversorgung, hiervon in besonderem Maße betroffen.

Zusätzlich verschärft wurde diese Situation durch die in der Regel für den gesamten Klinikbereich geltende Anpassung der „standard operating procedures" (SOP) auch für nichtstationäre $\mathrm{Pa}$ tienten und die weitgehende Einschränkung des Patienten- und Besucherstroms in den Krankenhäusern, die eine ersatzweise Verlagerung des Schwerpunkts der Patientenversorgung in den ambulanten Bereich unmöglich machte.

Darüber hinaus wurde die ophthalmologische Versorgung durch den fachfremden Einsatz von augenmedizinischem Personal in den vielerorts $\mathrm{zu}$ Beginn der Pandemie eingerichteten Infektionsambulanzen und Bereichen zur Aufrechterhaltung der COVID-19Versorgungskapazität sowie die in Kliniken der Maximalversorgung mitunter praktizierte Teilung der Ärzteteams zur Gewährleistung einer personellen Reserve erheblich eingeschränkt. Basierend auf den derzeit zur Verfügung stehenden Informationen und Daten, gibt dieser Artikel einen Überblick über die Strate- gien und Maßnahmen bettenführender Augenkliniken während der ersten Wochen der Pandemie.

\section{Präventive Maßnahmen und Infektionsschutz}

Die Vermeidung von direktem Personenkontakt bzw. die Einhaltung von Mindestabständen gilt weltweit als eine der wichtigsten Maßnahmen zur Verhinderung der Verbreitung von SARS-CoV-2 (- Abb. 1). Ziel dieser Maßnahme ist v. a., einen raschen Anstieg der Zahl der SARS$\mathrm{CoV}$-2-Infektionen in der allgemeinen Bevölkerung $\mathrm{zu}$ verhindern und damit die Gefahr des Auftretens von COVID19-Fällen mit schwerem Verlauf möglichst gering $\mathrm{zu}$ halten, um $\mathrm{zu}$ jedem Zeitpunkt eine intensivmedizinische Behandlung dieser Patienten gewährleisten zu können und gleichzeitig die Gefährdung von Risikogruppen durch SARSCoV-2 zu vermeiden [8].

Die Aufrechterhaltung einer ophthalmologischen Patientenversorgung bei gleichzeitiger Minimierung des Infektionsrisikos stellte die Kliniken vor völlig neue Herausforderungen, die eine Neuanpassung der SOP sowie eine völlige Umgestaltung von Abläufen und Warte- 


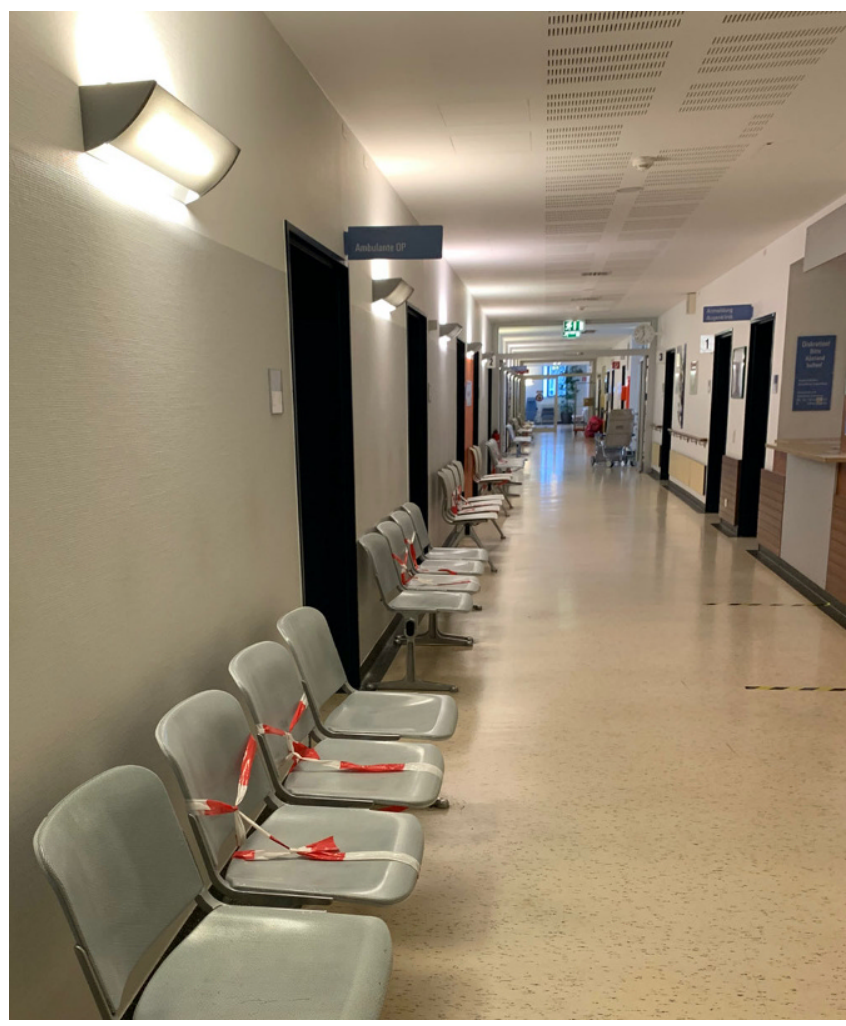

Abb. $1 \Delta$ Die Einhaltung von Mindestabständen gilt weltweit als eine der wichtigsten Maßnahmen zur Verhinderung der Verbreitung von SARS-CoV-2. Für die Aufrechterhaltung einer ophthalmologischen Patientenversorgung bei gleichzeitiger Minimierung des Infektionsrisikos werden Augenkliniken vor völlig neue Herausforderungen gestellt, die u. a. eine erhebliche Umgestaltung von Abläufen und Wartebereichen erfordern

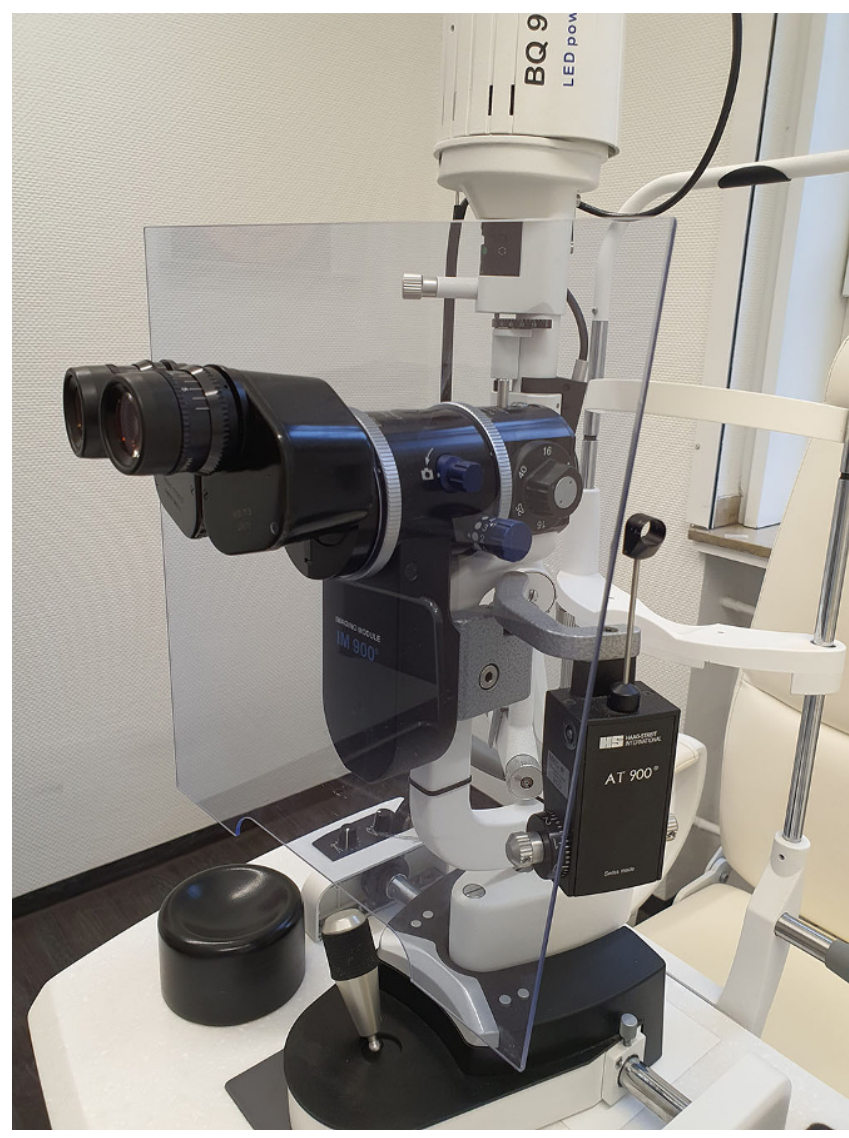

Abb. 2 A Infektionsprävention an der Spaltlampe: Plexiglasschutzschild. (Aus [21]) bereichen erforderte [9]. Als Teil dieser Strategie wurde in den bettenführenden Abteilungen vieler Kliniken der Maximalversorgung eine „Teilung der Teams“ (z. B. im wöchentlichen oder 14-tägigen Wechsel) vorgenommen, um im Falle der Ansteckung eines oder mehrerer Teammitglieder eine sofortige Quarantäne aller beteiligten Ärzte umsetzen zu können und durch den umgehenden Einsatz des "Reserveteams" eine kontinuierliche medizinische Versorgung zu gewährleisten. Mit der hierdurch entstehenden personellen Verknappung wurden Versorgungsengpässe zwar weiter verschärft, allerdings erforderte die im Vergleich zu anderen Fachgruppen besondere Gefährdung von Augenärzten auch eine besondere Aufmerksamkeit der Augenkliniken hinsichtlich geeigneter Hygienemaßnahmen, war doch eines der ersten Opfer unter dem medizinischen Personal in Wuhan jener Augenarzt, der vor dem Ausbruch der Pandemie gewarnt hatte [10, 11]. So hatte Anfang Februar 2020 die „American Academy of Ophthalmology" auf einen möglichen Zusammenhang zwischen dem Auftreten einer Konjunktivitis und 2019$\mathrm{nCoV}$ hingewiesen und entsprechende Vorsichtsmaßnahmen empfohlen. Es war vermutet worden, dass das Virus eine Konjunktivitis hervorrufen kann, die möglicherweise auch vor der Entwicklung einer respiratorischen Symptomatik auftritt und durch Tröpfcheninfektion über die Bindehaut übertragen werden kann.

Jüngere Publikationen zeigen jedoch, dass das Risiko einer Ansteckung durch Tränenflüssigkeit selbst bei COVID-19Patienten eher gering ist und auch die Häufigkeit des Auftretens einer Konjunktivitis nur bei etwa $1 \%$ liegt [12-14]. Hinzu kommt, dass das Angiotensin-Converting-Enzym 2 (ACE2), das für den Eintritt von SARS-CoV-2 in Körperzellen wahrscheinlich von Bedeutung ist, für den konjunktivalen Übertragungsweg wohl eher keine Rolle spielt. So konnten Lange et al. zeigen, dass u. a. der SARS-CoV-2Rezeptor ACE2 in der Bindehaut nicht in nennenswertem Maße auf mRNA- und Proteinebene synthetisiert wird, was eine konjunktivale Infektion mit SARSCoV-2 über diesen Mechanismus eher unwahrscheinlich macht [15].

Wie in einer kürzlich veröffentlichten Pressemitteilung der Deutschen Ophthalmologischen Gesellschaft berichtet, sollte Tränenflüssigkeit zwar dennoch als potenziell infektiöses Material behandelt werden, doch birgt für den Augenarzt v. a. die Nähe zwischen Patient und Untersucher, z.B. an der Spaltlampe, eine Ansteckungsgefahr durch Einatmung von Aerosolen aus der unmittelbaren Ausatemluft, weshalb für den Umgang mit potenziell Infizierten das Tragen eines Mund-Nasen-Schutzes (MSN) angeraten wurde $[11,12,16-20]$. Darüber hinaus sollten Plexiglasschutzschilder 
zur besseren Abschirmung bei Spaltlampenuntersuchungen zum Einsatz kommen (• Abb. 2; [21]).

\section{Ophthalmochirurgische Patientenversorgung während der Pandemie und „Krisenplan Ophthalmologische nicht- elektive Basisversorgung in bettenführenden Kliniken"}

Die Umstellung der Krankenhäuser auf die Herausforderungen der COVID-19Pandemie und die Fokussierung auf eine ausschließliche Versorgung von Notfällen und dringlichen Fällen stellte die Augenkliniken an bettenführenden Häusern in einem bisher nicht gekannten Ausmaß vor die Frage, welche Eingriffe aus ophthalmologischer Indikation wie dringlich durchgeführt werden sollten und in welchem Umfang eine Versorgung ophthalmologischer Patienten fortgesetzt werden kann [22-25].

Um den mit anderen operativen Fachrichtungen um Betten und OPKapazität konkurrierenden bettenführenden Augenkliniken eine einheitliche Priorisierung und Planbarkeit $\mathrm{zu}$ ermöglichen, wurde auf Initiative der Vereinigung Deutscher Ophthalmologischer Chefärzte (DOCH) und in Abstimmung mit den Vorständen der Retinologischen Gesellschaft (RG), der Vereinigung Ophthalmologischer Lehrstuhlinhaber (VOL) und der Deutschen Ophthalmologischen Gesellschaft (DOG) ein Krisenplan für eine nichtelektive ophthalmologische Basisversorgung erstellt, wobei die genannten Indikationen und Dringlichkeiten als grundsätzliche Empfehlungen aufzufassen sind, die im individuellen Fall und abhängig von der Ressourcenlage abgewogen werden müssen (• Abb. 3).

Ein ungefähres Bild der Situation und Vorgehensweise während der ersten Phase der Pandemie ergibt sich aus den Berichten der Augenkliniken der DOCH zur Erstellung des Krisenplans sowie aus einer von der VOL unter den Universitäts-Augenkliniken durchgeführten Umfrage. Ergebnisse lagen von 28 der 37 universitären Abteilungen vor. Danach war die ophthalmologische Versorgung von März bis April 2020

Ophthalmologe 2020 · 117:652-658 https://doi.org/10.1007/s00347-020-01162-x

(c) Springer Medizin Verlag GmbH, ein Teil von Springer Nature 2020

L.-O. Hattenbach · T. Reinhard · P. Walter · J. Roider · N. Feltgen · L. Hesse · J. Schrecker ·
N. Eter · Vereinigung Ophthalmologischer Lehrstuhlinhaber

Krisenstrategien der Kliniken während der Pandemie

Zusammenfassung

Hintergrund. Die SARS-CoV-2-Pandemie hat weltweit zu erheblichen Einschränkungen der medizinischen Versorgung geführt. Wir beschreiben bisherige Entwicklungen sowie Maßnahmen, die von März bis Mai 2020 zur Aufrechterhaltung der Versorgung ophthalmologischer Patienten an bettenführenden Augenkliniken beigetragen haben.

Methoden. Literaturrecherche über PubMed, eigene Daten, klinikübergreifende Umfrage. Ergebnisse. Durch die rasche Umsetzung von Hygienemaßnahmen und Anpassung der "standard operating procedures" (SOP) zur Minimierung des Infektionsrisikos sowie die Fokussierung auf Notfälle und dringliche Indikationen bei gleichzeitiger Aussetzung elektiver Behandlungen konnte eine kontinuierliche Versorgung ophthalmologischer Patienten aufrechterhalten werden.
Schlussfolgerung. Trotz der Herausforderung einer erheblichen Verlagerung medizinischer Ressourcen während der SARS-CoV-2Pandemie sind medizinisch dringlich notwendige ophthalmologische Behandlungen an Kliniken der Maximalversorgung gewährleistet. Anhand derzeit verfügbarer Daten kann allerdings nicht ausgeschlossen werden, ob es während der Pandemie auch zu Verzögerungen bei der Behandlung von Notfallpatienten gekommen ist.

Schlüsselwörter

SARS-CoV-2-Pandemie $\cdot$ Kliniken der Maximalversorgung $\cdot$ Hygienemaßnahmen . "Standard Operating Procedures" (SOP) . Notfallversorgung

\section{Crisis management strategies of hospitals during the pandemic}

\section{Abstract}

Background. The SARS-CoV-2 pandemic has led worldwide to substantial limitations in healthcare systems. This article describes the recent developments and measures from March through May 2020, which have contributed to the maintenance of ophthalmological care at in-patient departments of ophthalmology.

Methods. PubMed literature search, own data, interhospital survey.

Results. The rapid implementation of infection and hygiene control measures and adaptation of standard operating procedures (SOP) to minimize the risk of infection, along with prioritized urgent and emergency care combined with postponement of elective procedures enabled the continuous care of ophthalmological patients. Conclusion. Despite the challenge of a significant shift of medical resources during the SARS-CoV-2 pandemic, medically urgently necessary ophthalmological treatments are continuously provided by maximum care clinics; however, based on currently available data, it cannot be ruled out whether treatment of emergency patients was delayed during the pandemic.

\section{Keywords}

SARS-CoV-2 pandemic - Maximum care clinics - Hygiene control measures - Standard operating procedures (SOP) - Emergency care fast ausschließlich auf Notfälle, medizinisch dringlich indizierte Leistungen oder Kontrolluntersuchungen, aufgeschobene Behandlungen von Patienten mit zunehmender Dringlichkeit (z.B. Tumor mit Wachstum) und Patienten mit Schmerzsymptomatik oder Leidensdruck begrenzt. Geplante Operationen von Elektivpatienten erfolgten in dieser Zeit nicht. Elektive Eingriffe wurden neben nosokomialer Infektionsgefahr auch aufgrund der gesteigerten Betten- reservierung für COVID-19-Patienten ausgesetzt.

Während in den meisten Augenkliniken Medizinische Fachangestellte (MFAs) z.T. in den Zwangsurlaub geschickt wurden und ein Teil der Mitarbeiter über „Home Office“ mit der Klinik verbunden war, wurden AugenMFAs auch kurzfristig mit oder ohne vorherigem Training in Intensivmedizinbereichen oder anderen COVID-19assoziierten Bereichen (Virologie, Co- 


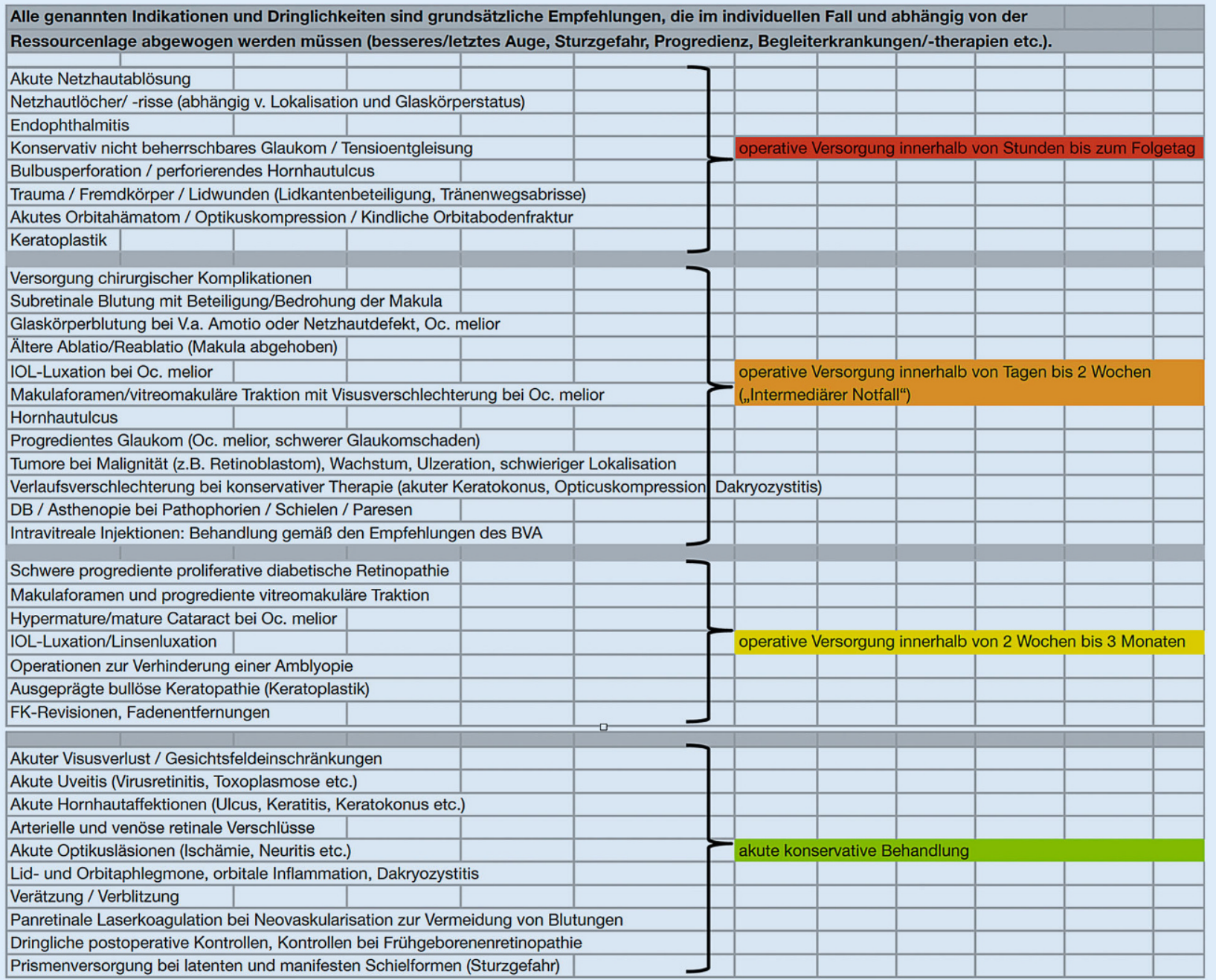

Abb. 3 \ Während der initialen Phase der Pandemie wurde auf Initiative der Vereinigung Deutscher Ophthalmologischer Chefärzte $(D O C H)$ und in Abstimmung mit den Vorständen der Retinologischen Gesellschaft $(R G)$, der Vereinigung Ophthalmologischer Lehrstuhlinhaber (VOL) und der Deutschen Ophthalmologischen Gesellschaft (DOG) ein Krisenplan für eine nicht-elektive ophthalmologische Basisversorgung erstellt. Die genannten Indikationen und Dringlichkeiten sind dabei als grundsätzliche Empfehlungen aufzufassen, die im individuellen Fall und in Abhängigkeit von der Ressourcenlage abgewogen werden müssen. (Mit freundl. Genehmigung der Autoren)

rona-Test-Ambulanzen, internistische Stationen) eingesetzt.

Insgesamt war in den meisten Kliniken ab Ende April/Anfang Mai ein schrittweiser Übergang in den Normalbetrieb feststellbar, allerdings mit zusätzlichen präventiven Maßnahmen wie Rachenabstrichen bei ambulant oder stationär zu operierenden Patienten, dem Verschieben von OP-Terminen um 2 Wochen bei symptomatischen Patienten oder einer präoperativen $\mathrm{Di}$ agnostik (Röntgen des Thorax, CT) bei zeitkritischen Patienten mit Atemwegssymptomatik.

Vielerorts wurden ab Mai auch aufgeschobene Operationen aus dem Lockdown durchgeführt bei z.T. eingeschränkter Kapazität an OP-Sälen und Personal zur stationären postoperativen Versorgung, da dieses zur Betreuung internistischer und infektiologischer Stationen eingesetzt wurde.

An allen befragten Kliniken erfolgte die Fortsetzung der an einen vorgegebenen Behandlungsplan gebundenen in- travitrealen operativen Medikamenteneingaben (IVOM) unter Einhaltung der Abstandsregeln im Wartebereich, Einsatz von MNS, Schutzvorrichtungen an den Untersuchungsgeräten sowie ohne Begleitpersonen im Wartebereich, allerdings in größeren zeitlichen Abständen und in einem um ca. $25-50 \%$ reduzierten Umfang, teilweise durch Absagen der zur Risikogruppe der Älteren zählenden Patienten selbst. 


\section{Leitthema}

Tab. 1 Mitglieder der Vereinigung Ophthalmologischer Lehrstuhlinhaber

\begin{tabular}{|c|c|c|c|c|c|c|}
\hline Titel & Vorname & Nachname & Abteilung & Institution 1 & Institution 2 & Ort \\
\hline Prof. Dr. & Gerd & Auffarth & & Univ.-Augenklinik & & Heidelberg \\
\hline Prof. Dr. & Karl Ulrich & Bartz-Schmidt & & Universitätsklinikum & $\begin{array}{l}\text { Department für Augenheil- } \\
\text { kunde }\end{array}$ & Tübingen \\
\hline Prof. Dr. & Nikolaos E. & Bechrakis & & Universitätsklinikum & Klinik für Augenheilkunde & Essen \\
\hline Prof. Dr. & Norbert & Bornfeld & $\begin{array}{l}\text { Abt. Erkr. Hinter- } \\
\text { abschnitt }\end{array}$ & Universitätsklinikum Essen & & Essen \\
\hline Prof. Dr. & Claus & Cursiefen & & Universitätsklinikum Köln & $\begin{array}{l}\text { Klinik und Poliklinik für } \\
\text { allgemeine Augenheilkunde }\end{array}$ & Köln \\
\hline Prof. Dr. med. & Burkhard & Dick & & Univ.-Augenklinik & & Bochum \\
\hline Prof. Dr. & Nicole & Eter & & $\begin{array}{l}\text { Universitätsaugenklinik } \\
\text { Münster }\end{array}$ & & Münster \\
\hline Prof. Dr. med. & Carsten & Framme & & Augenklinik MHH & & Hannover \\
\hline Prof. Dr. Dr. & $\begin{array}{l}\text { Thomas } \\
\text { Armin }\end{array}$ & Fuchsluger & & Universitätsklinikum Rostock & Augenklinik & Rostock \\
\hline Prof. Dr. & Gerd & Geerling & & Univ.-Augenklinik & & Düsseldorf \\
\hline Prof. Dr. Dr. h.c. & Franz & Grehn & & Univ.-Augenklinik & & Würzburg \\
\hline Prof. Dr. & Salvatore & Grisanti & & Univ.-Augenklinik & & Lübeck \\
\hline Prof. Dr. & Rudolf & Guthoff & & $\begin{array}{l}\text { Universität Rostock, Universi- } \\
\text { tätsmedizin }\end{array}$ & Augenklinik & Rostock \\
\hline Prof. Dr. & Horst & Helbig & & Univ.-Augenklinik & & Regensburg \\
\hline Prof. Dr. & Jost & Hillenkamp & & Universitäts-Augenklinik & & Würzburg \\
\hline Prof. Dr. & Hans & Hoerauf & & Univ.-Augenklinik & & Göttingen \\
\hline Prof. Dr. & Frank G. & Holz & & Univ.-Augenklinik & & Bonn \\
\hline Prof. Dr. & Jost B. & Jonas & & Seegartenklinik Heidelberg & Praxis für Augenheilkunde & Heidelberg \\
\hline Prof. Dr. & Antonia M. & Joussen & & $\begin{array}{l}\text { Charité - Universitätsmedizin } \\
\text { Berlin }\end{array}$ & $\begin{array}{l}\text { Campus Virchowklinikum } \\
\text { (CVK) }\end{array}$ & Berlin \\
\hline Prof. Dr. & Bernhard & Jurklies & & & & Essen \\
\hline Prof. Dr. & Thomas & Kohnen & & Klinik für Augenheilkunde & $\begin{array}{l}\text { Klinikum der J.W. Goethe-U- } \\
\text { niversität }\end{array}$ & $\begin{array}{l}\text { Frankfurt/ } \\
\text { Main }\end{array}$ \\
\hline Prof. Dr. & Friedrich $\mathrm{E}$. & Kruse & & Univ. Augenklinik & & Erlangen \\
\hline Prof. Dr. & Achim & Langenbucher & & $\begin{array}{l}\text { Medizinische Fakultät der } \\
\text { Universität des Saarlandes }\end{array}$ & Universität des Saarlandes & $\begin{array}{l}\text { Homburg/ } \\
\text { Saar }\end{array}$ \\
\hline Prof. Dr. Dr. & Chris Patrick & Lohmann & & Augenklinik & Klinikum rechts der Isar & München \\
\hline Prof. Dr. med. & Birgit & Lorenz & $\begin{array}{l}\text { Klinik und Polikli- } \\
\text { nik für Augenheil- } \\
\text { kunde }\end{array}$ & $\begin{array}{l}\text { Justus-Liebig-Universität } \\
\text { Gießen }\end{array}$ & $\begin{array}{l}\text { Universitätsklinikum Gie- } \\
\text { ßen und Marburg GmbH, } \\
\text { Standort Gießen }\end{array}$ & Gießen \\
\hline Prof. Dr. med. & Daniel & Meller & & Univ.-Augenklinik & & Jena \\
\hline Prof. Dr. & Christian & Ohrloff & & Univ.-Augenklinik & & $\begin{array}{l}\text { Frankfurt/ } \\
\text { Main }\end{array}$ \\
\hline Prof. Dr. & Norbert & Pfeiffer & & Univ.-Augenklinik & & Mainz \\
\hline Prof. Dr. & Lutz E. & Pillunat & & Augenklinik & $\begin{array}{l}\text { Augenklinik der Techni- } \\
\text { schen Universität }\end{array}$ & Dresden \\
\hline Prof. Dr. & Siegfried G. & Priglinger & & Universitäts-Augenklinik & & München \\
\hline Prof. Dr. & Thomas & Reinhard & & Klinik für Augenheilkunde & $\begin{array}{l}\text { Klinik für Augenheilkunde } \\
\text { des Universitätsklinikums } \\
\text { Freiburg }\end{array}$ & Freiburg \\
\hline Prof. Dr. & Johann & Roider & & Univ.-Augenklinik & & Kiel \\
\hline Prof. Dr. & André & Rosentreter & Augenheilkunde & Helios Universitätsklinikum & Wuppertal & Wuppertal \\
\hline Prof. Dr. Dr. & Stefan & Schrader & & Pius-Hospital Oldenburg & & Oldenburg \\
\hline Prof. Dr. & Berthold & Seitz & $\begin{array}{l}\text { Klinik für Augen- } \\
\text { heilkunde }\end{array}$ & $\begin{array}{l}\text { Universitätsklinikum des } \\
\text { Saarlandes UKS }\end{array}$ & & $\begin{array}{l}\text { Homburg/ } \\
\text { Saar }\end{array}$ \\
\hline Prof. Dr. med. & Walter & Sekundo & & Univ.-Augenklinik & & Marburg \\
\hline
\end{tabular}




\begin{tabular}{|c|c|c|c|c|c|c|}
\hline Titel & Vorname & Nachname & Abteilung & Institution 1 & Institution 2 & Ort \\
\hline Prof. Dr. & Martin & Spitzer & & $\begin{array}{l}\text { UKE Hamburg Klinik und } \\
\text { Poliklinik }\end{array}$ & $\begin{array}{l}\text { UKE Hamburg Klinik und Po- } \\
\text { liklinik für Augenheilkunde }\end{array}$ & Hamburg \\
\hline Prof. Dr. & Andreas & Stahl & & $\begin{array}{l}\text { Universitätsmedizin Greifs- } \\
\text { wald }\end{array}$ & $\begin{array}{l}\text { Klinik und Poliklinik für } \\
\text { Augenheilkunde }\end{array}$ & Greifswald \\
\hline Prof. Dr. med. & Hagen & Thieme & & $\begin{array}{l}\text { Universitätsklinikum Magde- } \\
\text { burg }\end{array}$ & Augenklinik & Magdeburg \\
\hline Prof. Dr. rer. nat. & Marius & Ueffing & & $\begin{array}{l}\text { Forschungsinstitut für Au- } \\
\text { genheilkunde }\end{array}$ & $\begin{array}{l}\text { Department für Augenheil- } \\
\text { kunde }\end{array}$ & Tübingen \\
\hline Prof. Dr. & Arne & Viestenz & & $\begin{array}{l}\text { Universitätsklinik und Polikli- } \\
\text { nik }\end{array}$ & $\begin{array}{l}\text { Universitätsklinik und Poli- } \\
\text { klinik für Augenheilkunde }\end{array}$ & Halle/Saale \\
\hline Prof. Dr. & Peter & Walter & & $\begin{array}{l}\text { Klinik und Poliklinik für Au- } \\
\text { genheilkunde }\end{array}$ & & Aachen \\
\hline Prof. Dr. & Peter & Wiedemann & & Univ.-Augenklinik & & Leipzig \\
\hline
\end{tabular}

\section{Ambulante ophthalmologische Versorgung}

Die allgemeinen Beschränkungen des Zugangs zu Kliniken mit starker Reduktion der Einbestellung von Patienten, dem Zugangsverbot von Begleitpersonen und Umsetzung der Hygieneregeln mit z.T. deutlich verlängerten Wartezeiten, aber auch die Furcht vieler Patienten vor Ansteckung im Krankenhaus als „CoronaHotspot" führten zu einem deutlichen Rückgang der Patientenzahlen in den Klinikambulanzen [26, 27]. Nach den Ergebnissen der Umfrage der VOL sowie Berichten aus den Kliniken der DOCH waren Notfälle und dringende Zuweisungen auf bis $\mathrm{zu} 30 \%$ der üblichen Fallzahl reduziert. Behandelt wurden ausschließlich Notfälle sowie individuell nach Dringlichkeit ausgewählte Patienten oder Patienten, die nach Verschieben des ursprünglichen Termins dringlich versorgt werden mussten, z. B. zur Amblyopiebehandlung oder Behandlung einer diabetischen Retinopathie.

Nachdem im März und April die ambulante Behandlung ophthalmologischer Patienten weitgehend auf eine nach der Dringlichkeit geordnete Versorgung beschränkt war, wurden in den meisten Kliniken ab Ende April bis Anfang Mai umfangreiche Hygienemaßnahmen als Voraussetzung für die Rückkehr der Ambulanzen zum Normalbetrieb umgesetzt. Hierzu zählen u.a. die Berücksichtigung von Abstandsregeln, das Tragen von Mund-Nase-Schutz (MNS) für Patienten und Mitarbeiter sowie ein Zugangsverbot für Begleitpersonen bzw. die Begrenzung auf eine Begleitperson bei Kindern und anderen bedürftigen Patienten.

Wesentliche Bestandteile der Maßnahmen waren darüber hinaus eine zeitversetzte Einbestellung, eine feste Sitzplatzzuordnung in den Wartezonen sowie die Untersuchung von SARS-CoV2-positiven Patienten in einer interdisziplinären COVID-Ambulanz.

Der Umfang der ambulanten Patientenversorgung in den Augenkliniken war in diesem Zeitraum allerdings durch die begrenzte Anzahl an Warteplätzen und Behandlungszimmern mit Gewährleistung eines ausreichenden Abstands auf etwa $50-60 \%$ des Normalbetriebes weiterhin limitiert. Der Einsatz telemedizinischer Lösungen spielte, im Gegensatz $\mathrm{zu}$ einer raschen und umfassenden Umstellung von Lehr- und Fortbildungsveranstaltungen auf Online-Formate, in den ersten Monaten der Pandemie keine wesentliche Rolle [28].

\section{Fazit}

Die SARS-CoV-2-Pandemie hat im März bis Mai 2020 zu massiven Einschränkungen der Versorgung ophthalmologischer Patienten und insbesondere nichtdringlichen ambulanten wie stationären chirurgischen Eingriffen und Behandlungen geführt.

Insgesamt konnte durch eine rasche Anpassung der SOP und Umsetzung der erforderlichen hygienischen Maßnahmen eine Grundversorgung für oph- thalmologische Notfälle und dringliche Behandlungen an den bettenführenden Hauptabteilungen erfolgreich aufrechterhalten und damit die Herausforderungen der Pandemie gut bewältigt werden.

Inwieweit der "Krisenmodus" der ophthalmologischen Versorgung auch zu einer lückenhaften („Corona-Angst“) oder verzögerten Behandlung von Notfallpatienten geführt hat, kann erst mit dem Vorliegen entsprechender Daten untersucht werden. Eine Umfrage der Kommission Sektorenübergreifende Augenheilkunde als gemeinsame Kommission von DOG und BVA hat eine onlinebasierte Umfrage erstellt, mit der ein umfassender Überblick zu den Auswirkungen der SARS-CoV-2-Pandemie auf die ophthalmologische Versorgung in Deutschland ermöglicht werden soll. Die Erkenntnisse dieser Umfrage sollen dazu beitragen, die Bedeutung der Aufrechterhaltung der Patientenversorgung in unserem Fach hervorzuheben und auf zukünftige Herausforderungen besser vorbereitet zu sein.

\section{Korrespondenzadresse

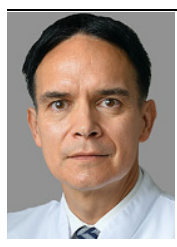 \\ Prof. Dr. Lars- \\ Olof Hattenbach, F.E.B.O \\ Augenklinik, Klinikum Ludwigshafen \\ Bremserstr. 79, 67063 Lud- wigshafen, Deutschland Hattenbach.LO@klilu.de}




\section{Einhaltung ethischer Richtlinien}

Interessenkonflikt. L.-O. Hattenbach, T. Reinhard, P. Walter, J. Roider, N. Feltgen, L. Hesse, J. Schrecker und $N$. Eter geben an, dass kein Interessenkonflikt besteht.

Für diesen Beitrag wurden von den Autoren keine Studien an Menschen oder Tieren durchgeführt. Für die aufgeführten Studien gelten die jeweils dort angegebenen ethischen Richtlinien.

\section{Literatur}

1. Ralli M, Minni A, Candelori F et al (2020) Effects of COVID-19 pandemic on otolaryngology surgery in Italy: the experience of our university hospital. Otolaryngol Head Neck Surg. https://doi.org/10. 1177/0194599820928970

2. Taha MA, Hall CA, Rathbone RF et al (2020) Rhinologic procedures in the era of COVID19: health-care provider protection protocol. Am J Rhinol Allergy. https://doi.org/10.1177/ 1945892420927178

3. Gupta A, Arora V, Nair D et al (2020) Status and strategies for the management of head and neck cancer during COVID-19 pandemic: Indian scenario. Head Neck. https://doi.org/10.1002/hed. 26227

4. Boettler T, Newsome PN, Mondelli MU (2020) Care of patients with liver disease during the COVID-19 pandemic: EASL-ESCMID position paper. JHEP Rep 2(3):100113

5. CovidSurg Collaborative, Nepogodiev D, Bhangu A (2020) Elective surgery cancellations Due to the COVID-19 pandemic: global predictive modelling to inform surgical recovery plans. Br J Surg. https:// doi.org/10.1002/bjs.11746

6. Argenziano M, FischkoffK, Smith CR (2020) Surgery scheduling in a crisis. N Engl J Med 382:e87

7. Diaz A, Sarac BA, Schoenbrunner AR et al (2020) Elective surgery in the time of COVID-19. Am J Surg. https://doi.org/10.1016/j.amjsurg.2020.04.014

8. Arons MM, Hatfield KM, Reddy SC (2020) Presymptomatic SARS-coV-2 infections and transmission in a skilled nursing facility. $\mathrm{N}$ Engl J Med 382:2081-2090

9. Glauser W (2020) Proposed protocol to keep COVID-19 out of hospitals. CMAJ 192(10):E264-E265

10. Green A (2020) Li Wenliang. Lancet 395(10225):1

11. Li OJP, Shantha J, Wong TY (2020) Preparedness among ophthalmologists: during and beyond the COVID-19 pandemic. Ophthalmology 127(5):569-572

12. Xia J, Tong J, Liu M et al (2020) Evaluation of coronavirus in tears and conjunctival secretions of patients with SARS-CoV-2 infection. J Med Virol 92:589-594

13. Seah IYJ, Anderson DE, Kang AEZ et al (2020) Assessing viral shedding and infectivity of tears in Coronavirus disease 2019 (COVID-19) patients. Ophthalmology. https://doi.org/10. 1016/j.ophtha.2020.03.026

14. Guan WJ, Ni ZY, Hu Y, Liang WH et al (2020) Clinical characteristics of coronavirus disease 2019 in China. N Engl J Med 382(18):1708-1720

15. Lange C, Wolf J, Auw-Haedrich C et al (2020) Expression of the COVID-19 receptor ACE2 in the human conjunctiva. J Med Virol. https://doi.org/ 10.1002/jmv.25981

16. Lim LW, Yip LW, Tay HW et al (2020) Sustainable practice of ophthalmology during COVID-19: challenges and solutions. Graefes Arch Clin Exp Ophthalmol 21:1-10

17. Breazzano MP, Shen J, Abdelhakim AH et al (2020) New York city COVID-19 resident physician exposure during exponential phase of pandemic J Clin Invest. https://doi.org/10.1101/2020.04.23. 20074310

18. Van Gerven L, Hellings PW, Cox T et al (2020) Personal protection and delivery of rhinologic and endoscopic skull base procedures during the COVID-19 outbreak. Rhinology 58(3):289-294. https://doi.org/10.4193/Rhin20.119

19. Arrigo A, Aragona E, Parodi MB et al (2020) Ophthalmology and SARS-coV-2: blind toward those who fight blindness? Eur J Ophthalmol. https://doi.org/10.1177/1120672120929961

20. Loon SC, Teoh SC, Oon LL et al (2004) The severe acute respiratory syndrome coronavirus in tears. $\mathrm{Br}$ J Ophthalmol 88(7):861-863

21. Heiduschka P, Lauermann JL, Bosche FD, Eter $\mathrm{N}$ (2020) Ein Schutz gegen die Tröpfcheninfektion an der Spaltlampe. Sicherheit für die Augenärzte UND die Patienten. Ophthalmologe 117(4):296-297

22. Karampelias V, Spanidis Y, Kehagias I (2020) Surgical practice and operative surgical strategies during the COVID-19T pandemic: a commentary. Ann Med Surg 55:47-48

23. Skalet AH, Allen RC, Shields $C L$ et al (2020) Considerations for the management and triage of ocular oncology cases during the COVID-19 pandemic. Ocul Oncol Pathol 6(3):1-4

24. Wasser LM, Assayag E, Tsessler M et al (2020) Response of ophthalmologists in Israel to the novel coronavirus (2019-nCoV) outbreak. Graefes Arch Clin Exp Ophthalmol 28:1-8

25. Korobelnik JF, Loewenstein A, Eldem B et al (2020) Guidance for anti-VEGF Intravitreal Injections During the COVID-19 Pandemic. Graefes Arch Clin Exp Ophthalmol 258:1149-1156

26. Lazzerini M, Barbi E, Apicella A (2020) Delayed access or provision of care in Italy resulting from fear of COVID-19. Lancet Child Adolesc Health 4(5):e10-e11

27. Yıldırım M, Geçer E, Akgül Ö et al (2020) The impacts of vulnerability, perceived risk, and fear on preventive behaviours against COVID-19. Psychol Health Med 3:1-9

28. Saleem SM, Pasquale LR, Sidoti PA et al (2020) Virtual ophthalmology: telemedicine in a Covid-19 era. Am J Ophthalmol. https://doi.org/10.1016/j. ajo.2020.04.029
Wie sich unser Gehirn Räume merkt

Das Gehirn konstruiert mentale Karten der Umwelt aus den Erfahrungen unserer Sinne - so können wir uns orientieren, uns erinnern, wo etwas passiert ist, und planen, wohin wir als Nächstes gehen. Forscher des Max-Planck-Instituts für Kognitions- und Neurowissenschaften in Leipzig und des Kavli Institute for Systems Neuroscience in Trondheim haben nun ein neues Computermodell entwickelt, das unserem Gehirn direkt dabei zuschauen kann, wie es sich orientiert und sich etwas merkt.

Sie konnten zeigen, dass neu gebildete Erinnerungen einen direkten Einfluss darauf haben, wie unser Gehirn die Umwelt abbildet. Je besser wir unsere Umgebung kennen, desto weniger Informationen müssen neu integriert werden. Dies wirkt sich direkt auf unsere Hirnaktivität aus und ist messbar.

Um eine breite Perspektive auf das menschliche Gehirn zu erhalten, verwendeten die Wissenschaftler funktionelle Magnetresonanztomographie. Die Studienteilnehmer lagen in einem 7-Tesla-MRT-Scanner und navigierten in einer virtuellen Arena mithilfe einer Tastatur: Sie merkten sich die Position von versteckten Objekten. „Um zu verstehen, wie sich unsere Teilnehmer bei dieser Aufgabe orientierten, haben wir analysiert, wie ihre Hirnaktivität zu jedem Zeitpunkt widerspiegelte, in welche Richtung sie sich bewegten.,' erklärt Nau.

Die neuartige Analysemethode ermöglichte es, die menschliche Richtungswahrnehmung in noch nie dagewesener Detailgenauigkeit zu vermessen. Die Ergebnisse deuten darauf hin, dass die neuronale Kartenbildung Einfluss darauf hat, wie das gesamte Netzwerk an Regionen im Gehirn Informationen verarbeitet, die wir gerade aus unserer Umwelt ableiten.

Originalpublikation: $\mathrm{Nau}, \mathrm{M}$. et al. Behavior-dependent directional tuning in the human visual-navigation network. Nat Commun 11, 3247 (2020). https://doi.org/10.1038/s41467-020$17000-2$

Quelle: Max-Planck-Institut für Kognitions- und Neurowissenschaften Leipzig: www.mpg.de [26.06.2020] 